\title{
Grafología Científica: La Muestra de Escritura como Test Psicológico Proyectivo*
}

\section{Scientific Graphology: Writing Sample as a Projective Test}

\author{
Tamara Rojas Weisser**
}

\begin{abstract}
Resumen
El siguiente artículo consiste en una reflexión sobre la fundamentación teórica de la Grafología, estableciendo que su objeto de estudio, la escritura, se constituye en un acto de proyección de la psique de quien escribe. Se propone la existencia de un Factor $\mathrm{T}$ a la base del proceso, una suerte de patrón constante y repetitivo que hace estable al acto escritural. Este factor T daría la base para considerar las fluctuaciones de la onda gráfica como reflejos de contenidos inconscientes, traducibles a una interpretación de rasgos profundos de personalidad. Por otra parte, también se revisa la metodología de análisis y los alcances clínicos de la técnica en orientación vocacional y profesional.
\end{abstract}

Palabras Clave: Grafología, Escritura, Factor T

\begin{abstract}
The following article consists on a reflection about the theoretical foundations of Graphology, establishing that its study object, handwriting, is a projection act of the psyche from the one who writes. The existence of a $\mathrm{T}$ Factor is proposed, being a part of the basics for this process, a sort of constant and repetitive pattern that makes the act of handwriting become stable. This T Factor gives the basic to consider the fluctuations of handwriting such as reflections from unconscious contents, which could be translated into an interpretation of deep features of personality. Moreover, the methodological analysis of handwriting is treated, as well as the clinical significances of the test in vocational and professional direction.
\end{abstract}

Key Words: Graphology, handwriting, T Factor.

* Agradecimiento a Ps. Elisabeth Wenk Wehmeyer, por su crítica constructiva en la redacción formal de este artículo.

** Psicóloga, Universidad de Chile. tamararojas_weisser@hotmail.com. 


\section{Introducción}

130 años de estudios científicos sobre las escrituras de diferentes personas a través de las épocas, en las cuales han participado psicólogos, psiquiatras, médicos, fisiólogos y filósofos entre otros profesionales, gestaron la construcción de un instrumento que goza actualmente de gran reputación y validez en Europa, especialmente en España y Francia ${ }^{1}$.

Si bien la grafología es un método con múltiples aplicaciones en los distintos campos de las ciencias y profesiones ${ }^{2}$, para los psicólogos cobra especial importancia e interés en cuanto descriptor de características de personalidad, especialmente desde que Max Pulver (1928) introdujera el psicoanálisis como fundamentación teórica. En ese momento, hito crucial, muchos psicólogos europeos se interesaron en profundizar acerca de la riqueza de contenidos inconscientes que podía reflejar el acto de escribir y dibujar, comenzando una serie de estudios psicológicos sobre el tema. Esto derivó no sólo en el desarrollo de las pruebas de la familia gráfica, que se basan en leyes o principios grafológicos, como son por ejemplo, el test del árbol de Koch, el test de la familia y la figura humana, sino también en el reconocimiento de la grafología como un test psicológico proyectivo del mismo nivel, comple- jidad y calidad de los tests de Zulliger, Lüscher y Rorschach, que los psicólogos chilenos consideran pruebas efectivas en el diagnóstico de estructura de personalidad.

En Chile en cambio, la Grafología no ha corrido igual suerte. Sufrió un prolongado período de marginación, relegada al campo del esoterismo, donde lamentablemente quedó atrapada durante muchos años. Esto, debido a que las personas que profesaban su conocimiento no presentaban la necesaria formación científica en las ciencias de la conducta humana, que derivó en un mal uso del instrumento. Muchas veces se malinterpretaron los signos, o se utilizaba como un medio para predecir el porvenir. No obstante, en la actualidad la Grafología ha comenzado a implementarse de una manera exponencial en el país, siendo cada día más requerida para la selección, promoción de candidatos en el ámbito profesional y en la orientación vocacional de jóvenes y adultos. Por lo mismo, cada día que transcurre, más colegas se muestran interesados en aprender a utilizar esta herramienta, incluso se sorprenden sobre el grado de efectividad que se puede alcanzar, sobre todo si se manejan con claridad los conceptos psicoanalíticos.

De hecho, existen dos investigaciones chilenas que pueden considerarse como impor-

\footnotetext{
1 Algunos ejemplos de estudios realizados por diferentes profesionales son los siguientes: Alfred Binet, en 1905, realizó un estudio para evaluar el grado de precisión de la grafología. El grado de acierto de cada grafólogo fue 92, 86, $83,80,68,66$ y 61\%. Binet concluyó que las diferencias de porcentaje dependía de la experiencia y pericia individual que tuviera el examinador. Gordon Allport y Philip Vernon, en los '30, psicólogos de la Universidad de Harvard, también estudiaron el análisis de escritura con técnicas estadísticas y trabajo experimental. Concluyeron que la escritura no era una actividad disociada de las complejidades de la personalidad, sino que de alguna manera se encontraba asociada en forma inherente con los determinantes subyacentes de la conducta. Wilhelm Preyer, Psicólogo infantil (a finales del siglo XIX y principios del siglo XX) fue el primero en descubrir que la escritura emana del cerebro, valorando la grafología como un instrumento de aplicación psicológica. Además comprobó que un escrito realizado con diferentes partes del cuerpo de una misma persona siempre presenta formas similares. Georg Meyer, Médico y Psiquiatra (1901) investigó las variaciones y perturbaciones orgánico-nerviosas en escritos de enfermos, descubriendo que los problemas de expresión obedecían a rasgos del carácter. Alfred Kanfer, Médico y grafólogo, (no hay data) descubrió que un análisis microscópico de la escritura en enfermos de cáncer manifiesta un deterioro más o menos importante en la coordinación neurovascular, lo cual era proporcional al avance de la enfermedad. Thea Stein Lewinson, en 1970, a través de su método grafométrico computacional, pudo descubrir 10 años antes que se declararan los estigmas esquizofrénicos latentes en 4 gemelas univitelinas por medio del estudio de sus cuadernos.

2 Se utiliza en medicina, criminología, psiquiatría, paleografía, etc.
} 
tantes precedentes para que la Grafología sea valorada seriamente por los profesionales en nuestro medio, de modo de debatir sobre sus alcances clínicos. El primero de estos estudios fue realizado en 1995, por la psicóloga Rebeca González, pionera en Chile en el estudio de escritura ${ }^{3}$. El segundo, realizado por la autora de este artículo en el año 2000. Ambos constituyen trabajos de título, cuyo fin último era experimentar con el test, evaluando su capacidad para determinar liderazgo en ejecutivos chilenos. El estudio de González comparó la escritura de ejecutivos chilenos con dotes de liderazgo y un grupo control. La segunda investigación, de tipo exploratoria, buscó determinar si la prueba, de presentar índices gráficos asociados a liderazgo, era capaz o no de arrojar un perfil de características de estos líderes y si este perfil resultante presentaría o no coincidencias con otros estudios contemporáneos sobre liderazgo chileno que emplearon otras técnicas para dicha definición ${ }^{4}$, además de comparar sus resultados con los encontrados en el primer estudio. Es interesante señalar que el perfil psicológico derivado de los índices grafológicos significativos encontrados resultó concordante con todas las investigaciones, y si bien el análisis de escritura no aportó nuevas características de personalidad que no estuvieran ya consideradas por las teorías e investigaciones más recientes sobre liderazgo, tuvo el valor de confirmar las características psicológicas atribuidas a este grupo de personas ${ }^{5}$.

El siguiente artículo tiene por objetivo entonces, introducir la grafología al debate académico a través de este ensayo sobre su fundamentación teórica, de acuerdo con la sistematización de la autora luego de haber analizado las propuestas de los psicólogos europeos expertos en la materia; por otra parte constituye un tema, que de acuerdo con su juicio quedó pendiente de desarrollar con mayor profundidad en su anterior trabajo. Finalmente también se llevan a cabo algunos comentarios finales sobre la grafología como prueba para uso e interpretación en el ámbito de la orientación profesional.

\section{Fundamentación Teórica: Una Grafología para Psicólogos}

Para poder valorar el proceso de análisis e interpretación que se realiza en grafología, es necesario primeramente remitirse a reflexionar sobre su objeto de estudio: la escritura.

Para la Grafología, la escritura es un acto complejo compuesto por una serie de oscilaciones rítmicas gatilladas a consecuencia de un proceso que implica un elevado compromiso neurofisiológico, que va desde la corteza cerebral (área sensoriomotora, área de Wernicke) pasando por la médula espinal, con la participación de los músculos del brazo, antebrazo y mano, además de la coordinación con la vista y los pensamientos devenidos conscientes que se estampan en la hoja mientras se escribe. El sustrato neurofisiológico a la base del acto explica sólidamente por qué la escritura es casi imposible de falsear; y también por qué es un acto complejo, pues implica no sólo un elevado compromiso corporal, sino además un proceso de aprendizaje previo (establecido en la escuela) como a su vez distintos factores de maduración psicobiológica. En este sentido, se puede aseverar que la escritura es un acto Voluntario, Automático y también una Proyección de la psique de quien escribe.

3 1995. Ver bibliografía para mayor información.

4 Estudios de liderazgo de la Pontificia Universidad Católica que utilizaron metodologías como observación de campo, encuestas, y bibliografía (Trabajos de Luis Enei 1988; Patricio Díaz 1989; Emilio Deik et al 1994; Paulina Martínez 1993, Bernardita Grove et al 1996). Cada estudio arrojó un perfil determinado de líder que fue compatible con el resultado del perfil psicológico elaborado a través del análisis de escritura. Para mayor información sobre éstos consultar la bibliografía. 5 Cfr.: Rojas et al. (2000). "Estudio exploratorio descriptivo de la personalidad de líderes ejecutivos chilenos a través del análisis de su escritura". Universidad de Chile. 
Para nuestra profesión, la escritura cobra valor cuando ésta se constituye en "razón gráfica $^{\prime \prime}{ }^{6}$ o acto psíquico, es decir, desde el momento en que el escribir tiene intención: comunicar algo a alguien o estampar pensamientos sobre algo o nosotros mismos. Del modo habitual y acostumbrado las personas se focalizan entonces en el análisis del contenido manifiesto, al igual como se acostumbra con el discurso oral de los pacientes. Se evalúa el mensaje escrito del escribiente como expresión de pensamientos devenidos conscientes, caracterizado por una coherencia argumentativa que da como resultado la hilación de un discurso con lógica consciente. Este mensaje muestra las representaciones-metas del escribir, es decir, la intencionalidad por la cual se escribe lo que se escribe, un discurso yoico en el que se evidencia explícitamente lo que se quiere comunicar al otro, que son las ideas y pensamientos. Para la psicoterapia, el contenido manifiesto se vuelve más interesante y aportativo cuando la hilación de estas ideas es interrumpida en forma reiterativa dentro del mensaje, presentándose los lapsus calamis o errores en el escribir. Estas interrupciones del discurso permiten esbozar algunas hipótesis vinculadas con el relato histórico dado por el paciente a través de las diversas sesiones, al igual como sucede con los lapsus lingüe del discurso oral. Se parte obviamente del supuesto que un monto de afecto escindido de su contenido reprimido ha sorteado los mecanismos de censura de la represión siendo liberado a la conciencia a través de otra agencia-representante investida por efecto del proceso de desplazamiento acontecido a nivel inconsciente, pues de alguna u otra manera presentaba algún tipo de conexión con el contenido pulsional reprimido original que la psique no está dispuesta a develar ${ }^{7}$. No obstante, la Grafología va más allá de este análisis, pues no se centra en desentrañar lo reprimido de lo inconsciente, sino que establece la escritura como un "acto polarizado", es decir "en medio del movimiento conciente formativo y el inconsciente deformador" ${ }^{\prime \prime}$. La escritura por tanto, proyectaría un contenido latente que es lo que el análisis de escritura estudia e interpreta psicológicamente.

El contenido latente estaría definido o determinado por la onda gráfica en sí misma o trazo estampado en la hoja en blanco. Así, no sólo cada retoque o tachadura, sino también cualquier desproporción en la onda, diferencias en la intensidad, magnitud, agilidad, calidad, tipo y estilo de enlace, forma, ordenamiento, etc., evidencia un registro de cómo se da esta suerte de pulsión "gráfica", la cual acontece en forma paralela a lo que es la razón gráfica. Es decir, el discurso consciente o mensaje explícito se deja de considerar como objeto de estudio y se analiza la onda gráfica como un dibujo que reflejaría contenidos inconscientes de nuestra psique vinculados a las percepciones sensoriales, procesos afectivos e intelectuales que caracterizarían a un individuo. Cualquier rigidez en el grafismo, irregularidad o regularidad, bizarramiento, personalización o apego excesivo a normas caligráficas, por ejemplo hablará tanto del tipo, el estilo como de la profundidad de la neurosis del paciente o si aquél presenta una psicopatología severa. Señalará el modo de organización de la psique, el modo de negociación que se da entre las instancias psíquicas, cuál gobierna sobre otra, cuánta fuerza yoica existe; describirá cómo es la adaptación del sujeto y qué tipo de tendencias prevalecen sobre otras, hacia dónde se dirige, cómo se dirige, si se satisface o se inhibe de modo adecuado o inadecuado la libido, estándose en condiciones de descifrar o traducir a características intelectuales, afectivas y sociales.

6 Término utilizado por Inarra, Dominique. 2001.

7 De acuerdo con lo que interpreta la autora de la lectura del capítulo de "La represión" de Sigmund Freud, 1990 (ver bibliografía).

8 Solange Pellat y Ludwig Klages, cit. en Xandró, M.,1994, pág. 29. 
La onda gráfica habla de una historia psíquica, una historia que, en cuanto acto consciente, tiene su complemento inconsciente. No sólo se lleva a cabo la evaluación de los lapsus calami, sino también de la dinámica pulsional que se gesta mientras se escribe. Cualquier alteración o anomalía, si no está relacionada con causas fisiológicas o neurológicas, será un índice interpretable en términos psicológicos. No debe olvidarse que la psique manifiesta sus contenidos inconscientes a través de diversas formas en el lenguaje verbal, paraverbal y corporal, aconteciendo lo mismo en la escritura, pues es otro acto de comunicación, es un registro de comportamiento. Si por ejemplo, cada pulsión de repetición habla de algo reprimido u olvidado que se lleva al acto, la repetición mecánica de la onda gráfica manifiesta algo que se está trazando (actuando) ya que es en el mensaje explícito donde la psique conciente concentra su atención y descuida la onda que se emite.

Si se observa la escritura propia y se la compara con la de otras personas, se podrá apreciar que cada escritura tiene sus características peculiares, reflejos de contenidos o rasgos psíquicos que comunican su existencia. Y si se observa detenidamente muestras de escrituras de nosotros realizadas en distintos intervalos de tiempo, también es posible darse cuenta que existe lo que esta autora ha decidido denominar "Factor $T$ ", un factor de constancia en la onda gráfica que le da identidad, independientemente de las circunstancias anímicas o de salud que se esté viviendo, las cuales permanecen trascendiendo el tiempo. Este factor $\mathrm{T}$ es una suerte de patrón repetitivo, constante y particular, que hace estable al acto y el que sostiene la razón por la cual la escritura puede ser estudiada en su gráfica motriz como una proyección de contenidos inconscientes, traducibles a una interpretación respecto de rasgos profundos de la personalidad. Por ejemplo, si un individuo cambia recurrentemente el tamaño de su escritura o la velocidad de sus trazos, ese es el manifiesto de su factor $\mathrm{T}$, a diferencia de una persona que denote una escritura siempre bien dibujada, con la misma velocidad o inclinación de letras: eso será lo característico de su factor $\mathrm{T}$ y lo diferenciará del otro. Justamente es este factor T, que se encontraría en la frontera de lo fisiológico y lo psíquico, una suerte de libido expresándose a través de la onda gráfica, como anteriormente se señaló entre el consciente formativo y el inconsciente deformador?.

Por lo tanto se puede aseverar, a modo de resumen, que lo inconsciente se percibe en el modo de configuración del discurso. En el caso del lenguaje oral todos los aspectos paraverbales, como tono de voz, rapidez de la expresión, emocionalidad, fuerza e intensidad de lo que se expresa, como también la lógica discursiva y sus accidentes o fluctuaciones, develan contenidos inconscientes reprimidos o no. En el caso del lenguaje escrito, la proyección del inconsciente acontece entonces en la fluctuación de la onda (se lo puede llamar lo "paraverbal" de la onda), que devela qué tipo de comportamiento se dibuja en la hoja en blanco. Los caminos adoptados por lo inconsciente no sólo se limitan a la lógica discursiva del lenguaje oral y los gestos corporales, sino también al modo o patrón del registro escrito. No debe olvidarse que lo inconsciente denota otra temporalidad y lógica de lo consciente, por lo cual busca vías diferentes de manifestación, no obstante complementarias y paralelamente contemporáneas a la emisión del consciente, las cuales deben ser interpretadas para lograr una exploración profunda de los estados internos.

9 Augusto Vels, en su obra "Escritura y Personalidad" 1997, ya comienza a hacer mención de esta cualidad en la onda gráfica la cual compara con un electroencefalograma, refiriéndose a que la grafología se focaliza en estudiar las fluctuaciones en intensidad, presión, forma, rapidez, etc., pero no se profundiza mayormente en el fenómeno mismo del Factor T ni tampoco en otorgarle una acuñación específica. 


\section{Definición de la Grafología}

Actualmente existen muchas definiciones en la bibliografía, pero se las puede resumir en: "Un test psicológico proyectivo, cuyo fin es describir las características de personalidad de un individuo a través de la observación, medición, análisis e interpretación de las manifestaciones que acontecen durante el proceso y dinámica del recorrido que el lápiz realiza en el decurso de una página en blanco, configurando con su tinta un trazo u onda gráfica propia, particular y diferente en cada persona".

\section{Metodología para la Interpretación}

¿Por qué la Grafología constituye un Test Proyectivo? En la actualidad el estado de conocimientos e investigaciones sobre el tema han determinado principios y leyes a la base de la técnica, estableciéndose un método de clasificación universal de los índices manifiestos en una grafía, con su respectiva medición, diagnóstico e interpretación.

Lo primero es respecto al instrumento, la página en blanco, la cual se considera, de acuerdo con Max Pulver, como el espacio y tiempo vitales ${ }^{10}$, donde el texto y la firma vendrían a gestar una estructura gráfica con determinada forma, espacio y movimiento ${ }^{11}$ que reflejaría el modo de estructuración de la personalidad frente a este estímulo desestructurante.

La forma, espacio y movimiento adoptados por cada escritura se divide para su estudio en los llamados "Aspectos Gráficos", cada uno de los cuales, en su conjunto, definen di- ferentes rasgos de personalidad. Dichos aspectos gráficos son: Tamaño, Orden, Forma, Inclinación, Continuidad, Presión, Velocidad, Dirección y Gestos Tipo (éstos se han resumido en la Tabla $N^{\circ}$ I que se entrega al final del presente artículo). Cada uno de ellos presenta su forma de tabulación específica y campo interpretacional, como una variada gama de índices y subíndices con una interpretación propia. Dichos aspectos se aplican tanto al Texto como a la Firma y la Rúbrica.

Para realizar la evaluación de los aspectos gráficos y proceder a la posterior interpretación psicológica del conjunto gráfico, se deben realizar las siguientes etapas:

1. Etapa Grafométrica: Consiste en la utilización del "Inventario de los Signos Gráfi$\cos ^{\prime \prime 12}$ que organiza y sistematiza la evaluación cuantitativa de los signos presentes en un escrito de acuerdo con los resultados que arrojan instrumentos específicos, como regla milimetrada, transportador de grado, lupa, transparencia de márgenes, etc.

2. Conversión de índices a Escritura Tipo: Una vez realizadas y promediadas las mediciones, se convierten a una Escritura Tipo, la cual está definida por requisitos que se deben cumplir dentro de la onda gráfica para dicha categoría ${ }^{13}$.

3. Jerarquización de los signos a Dominantes y Secundarios: Una vez definidas las escrituras tipo, se procede a dar peso a cada una de ellas, de acuerdo con su grado de presencia, frecuencia e impacto que tienen en

10 Max Pulver 1928, cit. en Vels, A., 1997a; Simón, J. J., 1992.

11 Gross y Rudolf Heiss, cit. en Vels, A., 1997(b), propusieron que la onda gráfica presenta tres grandes aspectos fundamentales, los cuales se considerarían como ejes fundamentales en el análisis de escritura, ya que permiten formarse una impresión general del escrito, dando la base para la interpretación de los demás componentes de la escritura.

12 Rojas W., Tamara, 2000. Creado por la autora para su trabajo anterior: Se trata del diseño de un sistema que no sólo permite evaluar la onda gráfica lo más exhaustivamente posible, sino también facilita la revisión y contrastación de cada medición.

13 Existen una serie de reglas y módulos que especifican el criterio de definición de la onda gráfica. Por ejemplo, para categorizar que una escritura es grande, el cuerpo medio de la onda debe medir entre 3,5 a 4,5 mm de altura, la cual tiene una serie de reglas para la medición. 
la onda gráfica. Existe un principio grafológico que sustenta esta condición, el cual señala que no se puede dar el mismo peso interpretativo a un indicador que aparece de forma esporádica en un grafismo, a otro que está presente de principio a fin, influyendo en forma preponderante en el comportamiento de la escritura.

4. Determinación del ambiente gráfico: Como los signos tienen un valor relativo, es decir, su interpretación está condicionada por el contexto en el cual se encuentran implicados, se debe establecer la positividad o negatividad del conjunto, el cual determinará el modo de interpretación psicológica a utilizar.

5. Determinación de competencias, aptitudes o rasgos de interés (acorde al objetivo del informe), a través de la determinación de matrices gráficas. Existe dentro de la escritura la posibilidad de detectar competencias específicas, las cuales se definen por agrupaciones de signos.

6. Elaboración del Informe: De acuerdo con el objetivo de la medición, se puede realizar una descripción general de rasgos de personalidad, focalizarse en competencias específicas, diagnosticar estructura de personalidad, etc. Se puede dividir por las áreas clásicas que se utilizan (intelectual, emocional, social) o realizar un informe dinámico.

\section{Aplicaciones en Orientación Vocacional y Profesional: Sus Alcances Clinicos}

El valor y alcance clínico que adquiere el análisis de escritura en la orientación vocacional de jóvenes y profesionales es de tipo diagnóstico, pudiendo utilizarse en una batería de pruebas. La escritura, ya explicada anteriormente, también puede ser interpretada para detectar las aptitudes profesionales de un sujeto, además de sus conflictivas psíquicas. De acuerdo con el comportamiento configurado por la onda, se pueden eva- luar los recursos que tiene un(a) joven, por ejemplo:

En inteligencia: Describir el nivel cultural o intelectual, la claridad de ideas, la velocidad de razonamiento, si presenta un pensamiento teórico, práctico, intuitivo o creativo.

En cualidades profesionales: Determinar el nivel de rendimiento, el grado de constancia, iniciativa, capacidad de decisión, el grado de organización-planificación, capacidad mnémica, aptitudes espaciales, sentido estético, liderazgo-aceptación de la autoridad, necesidad de apoyo, necesidad de reconocimiento, cantidad y calidad del trabajo, fiabilidad o integridad, aspiraciones personales, etc.

En comportamiento social: El grado de sociabilidad, el tipo de establecimiento de relaciones (extensivas-intensivas), excentricidad, independencia de criterios o apego a las normas, dependencia del grupo, tendencia al idealismo o realismo, capacidad de negociación, intransigencia, mordacidad, grado de espontaneidad o artificiosidad en el trato con el otro, obstinación, oposicionismo, sensualidad, disposición al cambio, desconfianza, egoísmo-altruismo, egocentrismo, objetividad-subjetividad de las relaciones, mitomanía, tendencia al gasto o al ahorro, vanidad-sencillez.

En factores emocionales: Si predomina el sentimiento o la razón, la Fuerza del Yo, el autocontrol, autoncepto alto o bajo, sentimientos de inferioridad o superioridad, optimismo-pesimismo, etc.

Agresividad y sexualidad: Determinar si se presenta una agresividad de tipo primaria o secundaria, hacia dónde se canaliza (hacía sí mismo o los demás).

Psicopatología: Si se presenta un cuadro neurótico, ya sea obsesivo o histérico; o una psicosis: paranoide, maníaco-depresiva, esquizofrenia, psicopatías, tendencia al suicidio. 
En síntesis, se puede deducir del comportamiento de la onda gráfica si existe una tendencia o no hacia las aptitudes artísticas, científicas, humanas, comerciales, o aquellas relacionadas con lo administrativo o contable: la clasificación, la ordenación, control y verificación de datos; o aquellas vinculadas con lo más técnico y /o manual (operativos).

\section{Comentarios Finales}

Los alcances de la Grafología aplicada de manera científica y profesional, son de orden diagnóstico. Su aplicación, por lo tanto, no sólo se limita al campo de la orientación profesional de jóvenes, sino también a la orientación profesional de adultos, muchas veces mal conducidos en sus elecciones de carrera. Se pueden redirigir sus potencialidades hacia campos de la profesión donde se requieran de las cualidades por ellos presentadas. La experiencia de la autora con el test le ha permitido darse cuenta en reiteradas oportunidades que la historia de frustraciones y fracasos vividas por algunos profesionales ha sido consecuencia de una errada postulación. Por diversas razones encauzaron sus vidas por vías que no les permitían desarrollar sus verdaderas destrezas. La aplicación del test les permitió, tal como una suerte de coaching, reformular sus metas y redirigirse a la búsqueda de cargos que estuvieran más en línea con sus características de personalidad y competencias presentadas en la grafía.

Respecto al Factor $\mathrm{T}$, se puede señalar que es un modo de acuñar un fenómeno que acontece en la onda gráfica, el cual se daría independiente y paralelamente a los índices que dan cuenta de los estados fisiológicos, físicos, estados emocionales y contenidos relacionados con aspectos reprimidos relacionados con la historia pasada o presente del escribiente. El Factor T sería una constante estable, permanente y repetitiva que da cuenta de fenómenos psíquicos que tratan de hacer manifiesto su existencia, y que en su conjunto darían comunicación de una estructura psíquica particular en cada individuo posible de traducir a rasgos de personalidad estables a través del tiempo, y por ende a considerar el análisis de escritura como un instrumento psicológico proyectivo.

Finalmente, la autora espera que este breve ensayo permita estimular el interés por la Grafología entre los colegas, para debatir abiertamente sobre el tema en un futuro, deseablemente en el corto plazo. 
Tabla I. "Aspectos Gráficos" 14

\begin{tabular}{|c|c|c|}
\hline $\begin{array}{c}\text { Nombre } \\
\text { del Aspecto }\end{array}$ & Breve Definición & Interpretación Psicológica asociada \\
\hline Dimensión & $\begin{array}{l}\text { Tamaño de la escritura en altura y } \\
\text { anchura como espacio ocupado por } \\
\text { los movimientos gráficos en cada } \\
\text { una de las } 5 \text { zonas del espacio. }\end{array}$ & $\begin{array}{l}\text { Autoestima y capacidad expansiva (confianza y } \\
\text { autonconfianza). Megalomanías, caracteres anales y } \\
\text { orales (Dimensión). Ârea de la personalidad donde } \\
\text { el escribiente tiene sus principales necesidades (Je- } \\
\text { rarquía). }\end{array}$ \\
\hline Orden & $\begin{array}{l}\text { Distribución, Disposición y Pro- } \\
\text { porción de la escritura. }\end{array}$ & $\begin{array}{l}\text { Grado de equilibrio de las tendencias. Ubicación } \\
\text { psicológica frente a las personas y el entorno. Or- } \\
\text { den y claridad en las ideas, los afectos y la conduc- } \\
\text { ta. Determinación del juicio de realidad para dife- } \\
\text { renciar a los cuadros psicóticos. }\end{array}$ \\
\hline Forma & $\begin{array}{l}\text { Modalidad de estructura y ejecu- } \\
\text { ción de las letras. }\end{array}$ & $\begin{array}{l}\text { Reflejo de la expresión modal de conducta; tipos de } \\
\text { actitudes adoptadas para la integración al orden so- } \\
\text { cial, grado de autenticidad, artificiosidad o excentrici- } \\
\text { dad. Determinación de niveles de neurosis, psicosis. }\end{array}$ \\
\hline Inclinación & $\begin{array}{l}\text { Orientación en grados de trans- } \\
\text { portador que las letras tienen ha- } \\
\text { cia la izquierda, derecha o verti- } \\
\text { cal del espacio }\end{array}$ & $\begin{array}{l}\text { Grado de vinculación afectiva que puede ir desde los } \\
\text { bloqueos o la insensibilidad, pasando por los carac- } \\
\text { teres oposicionistas, la desconfianza, la independen- } \\
\text { cia afectiva hasta la necesidad de contacto con los } \\
\text { demás (diferentes grados hasta la superficialidad de } \\
\text { las relaciones). Cuadros de tendencia histérica. }\end{array}$ \\
\hline Dirección & $\begin{array}{l}\text { Dirección en ascenso-descenso de } \\
\text { líneas, Orientación de los signos } \\
\text { y abreacción de letras. }\end{array}$ & $\begin{array}{l}\text { La dirección de líneas permite establecer la tenden- } \\
\text { cia al optimismo, pesimismo (cuadros depresivos, } \\
\text { deliriosos, o bipolares). } \\
\text { La orientación permite definir la actitud vital (ex- } \\
\text { trovertido-ambitendente-introvertido). } \\
\text { La abreacción señala el grado de sinceridad (abar- } \\
\text { cando ambos extremos). }\end{array}$ \\
\hline Continuidad & $\begin{array}{l}\text { Porcentaje de uniones entre le- } \\
\text { tras de una palabra. Y grado de } \\
\text { variación en los diversos aspec- } \\
\text { tos gráficos. }\end{array}$ & $\begin{array}{l}\text { Grado de unión, homogeneidad, perseverancia y } \\
\text { estabilidad en las ideas, en el modo de ser y hacer. } \\
\text { Detección de tendencias esquizoides, paranoides, } \\
\text { histéricas, obsesivas. }\end{array}$ \\
\hline Presión & Surco de tinta en el papel & $\begin{array}{l}\text { Valoración de la potencia de la libido que se } \\
\text { extrapola a lo intelectual, lo emocional, lo social y } \\
\text { laboral. También evidencia fallos en el estado de sa- } \\
\text { lud, debilidad de carácter, propensión a la fatiga, etc. }\end{array}$ \\
\hline Velocidad & $\begin{array}{l}\text { Rapidez con que los trazos se es- } \\
\text { criben }\end{array}$ & $\begin{array}{l}\text { Rapidez de los procesos mentales, de elaboración y } \\
\text { comprensión, como los grados de reflexión, intuición. } \\
\text { Detección de problemas de inteligencia, dificulta- } \\
\text { des de aprendizaje, problemas con la memoria, en- } \\
\text { tre otros. }\end{array}$ \\
\hline Gestos Tipo & $\begin{array}{l}\text { Aquellos que escapan al control } \\
\text { de la persona y que se correspon- } \\
\text { den con los signos accesorios al } \\
\text { mensaje o contenido principal. }\end{array}$ & $\begin{array}{l}\text { Diversas interpretaciones de acuerdo con el gesto } \\
\text { tipo. Abarca la capacidad atencional, dotes de man- } \\
\text { do y autoridad, dirección de la agresividad, preci- } \\
\text { sión y exactitud en las cosas, etc. }\end{array}$ \\
\hline
\end{tabular}

14 La tabla es un resumen de la sistematización propuesta por los españoles Augusto Vels, 1997; José Javier Simón, 1992; quienes a su vez se basaron en la propuesta francesa de Jules Crepieux-Jamin, uno de los padres de la Grafología Moderna. 


\section{Referencias Bibliográficas}

Curtis, Helena y Barnes, N. Sue. (1993). Biología. Madrid, España. Editorial Médica Panamericana. Quinta edición.

Deik, Emilio; Vergara Cristóbal. (1994). Perfil del Ejecutivo Chileno Exitoso. Memoria para optar al título de Ingeniero Civil de Industrias con mención en computación. Santiago, Chile. Pontificia Universidad Católica de Chile.

Díaz, Patricio. (1989). Perfil del Ejecutivo Chileno. Tesis para optar al grado de Magíster en Ciencias de la Ingeniería. Santiago, Chile. Pontificia Universidad Católica de Chile.

Inarra, Dominique. (2001). Pulsión y Razón Gráfica (traducción de Gabriela Yankelevich). Revista Acheronta No13. (www. Acheronta.org).

Echevarría, M. Elina. (1992). Grafología Infantil. Madrid, España. Editorial Edaf, S.A.

Enei, Luis. (1988). Liderazgo Ejecutivo en Chile. Tesis para optar al grado de Magíster en Ciencias de la Ingeniería. Santiago, Chile. Pontificia Universidad Católica de Chile.

Freud, Sigmund. (1990). Obras Completas. Buenos Aires, Argentina. Editorial Amorrortu, Volumen 14.

González, Rebeca. (1995). Estudio Descriptivo Comparativo de Indicadores Grafológicos en la escritura de ejecutivos. Tesis para optar al grado de Licenciada en Psicología. Santiago, Chile. Universidad Diego Portales.
Grove, B.; Pallamar, P.; Mehech, A. (1996). Liderazgo Ejecutivo en Empresas Chilenas: Un Perfil para los '90. Santiago, Chile. Pontificia Universidad Católica de Chile.

Hughes, A. (1970). Guía Práctica de Grafología. Madrid, España. Editorial Edaf, S.A.

Martínez, Paulina. (1993). Revisión Crítica de los aportes sobre Liderazgo y Efectividad en los últimos diez años. Tesis para optar al grado de Licenciado en Psicología. Santiago, Chile. Pontificia Universidad Católica de Chile.

Moracchini, Michel. (1995). ABC de la Grafología. Girona, España. Tikal Ediciones.

Rojas, Tamara et al. (2000) Estudio Exploratorio Descriptivo de la Personalidad de Líderes Ejecutivos chilenos de la región metropolitana a través del Análisis de Escritura. Memoria para optar al título de Psicólogo. Santiago, Chile. Universidad de Chile.

Simón, José Javier. (1992). ABC de la Grafología. España. Editorial Martínez de Roca S.A.

Vels, Augusto. (1997 a). Escritura y Personalidad. Barcelona, España. Editorial Herder S.A., Novena edición.

Vels, Augusto. (1997 b). Grafología Estructural y Dinámica. Barcelona, España. Editorial Herder, S.A., Segunda edición.

Xandró, Mauricio. (1997). Grafología Elemental. Barcelona, España. Editorial Herder S.A., Quinta edición. 